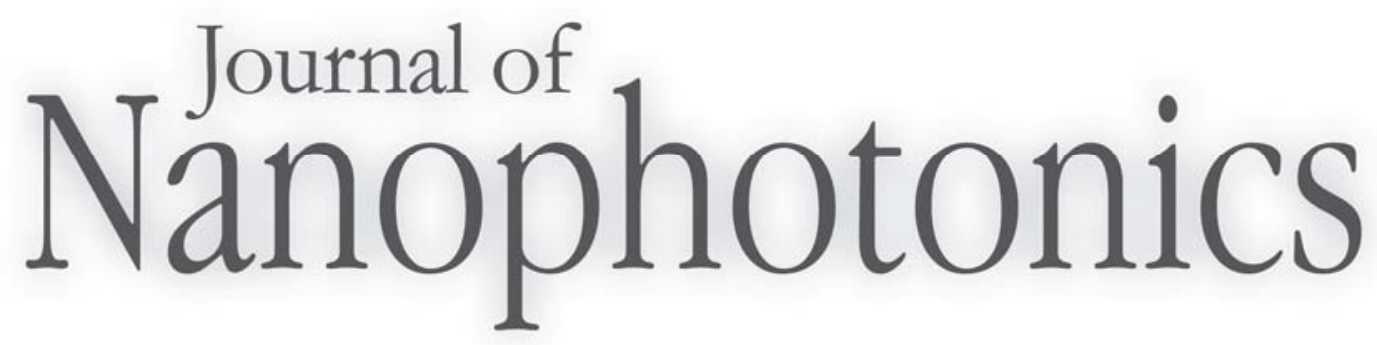

SPIEDigitalLibrary.org/jnp

\title{
Book Review: \\ Diamond Nanotechnology: Synthesis and Applications
}

Reviewed by William B. White 


\title{
BOOK REVIEW
}

\section{Diamond Nanotechnology: Synthesis and Applications}

\author{
James C. Sung and Jianping Lin, 252 pages, ISBN 978-981-4241-41-0, Pan Stanford,
} Singapore (2010), US\$149.00, hardcover.

Reviewed by William B. White, Pennsylvania State University, Materials Research Institute, University Park, PA 16802, wbw2@psu.edu



Diamond: the hardest natural substance known; a gemstone of great worth; an optical material of superior properties, and perhaps the semiconductor of the future. Certainly of all substances of interest to the materials scientist, diamond has the most romantic history. Diamond is elemental carbon with the carbon atoms arranged with each carbon surrounded by four other carbons in a perfect tetrahedral arrangement that produces a cubic crystal. For all of the crystallographic simplicity, the synthesis of diamond, the control of its properties, and the invention of applications have been supreme technological challenges. Thus, Sung and Lin have a great deal to talk about.

One might begin with the title. The book is not exclusively about nanodiamond technology. It covers much more than technology and nanodiamonds make up only a fraction of the discussion.

The first chapter discusses the origin of the elements, especially carbon, with some garbled physics of how carbon is synthesized in the stars. Sung proposes that diamonds are very common in the Universe, that there are stars with diamond cores, and that some planets, Neptune, for example, have showers of diamonds in their atmospheres. Then follows a wildly speculative chapter on the role of diamonds in the origin of life, the theory that life on Earth was seeded from space-RNA riding on nanodiamondsand the possible role of diamond as a template on which the complex precursors of life could have developed. Then there is the proposition that nanodiamonds could act as chips on which neuron cells could be built into a super brain. "The integration of living brain and material world may reveal the mind of God.” How's that for hubris?

The authors return to Earth beginning with chapter 3 on the properties of diamond, chapter 4 on the history of the high pressure synthesis of diamond, and chapter 6 on micron fines and nanodiamonds. Chapter 6 is particularly good with much useful technical detail on the particle size and shapes of diamonds used as abrasives. Particularly fascinating is chapter 7 on dynamite diamonds - the techniques for producing nanodiamonds by high explosives. The remaining chapters discuss the applications of diamond including lubricants, various biological applications, thermionics, photovoltaics, and coatings. The final chapter, with a jump from nano to macro, discusses the synthesis of gem-quality diamonds.

This is an oddly structured book and the intended audience is not obvious. Ten of the eleven chapters were written by J. C. Sung in a popular-almost stream-of-consciousness 
style. But there are many technical illustrations—photographs, diagrams, tables, and spectra. The sources of these illustrations are rarely cited. There are no references or even suggestions for further reading. It's an unfortunate omission. There are a great many interesting tidbits, mentioned in passing, for which I wish I could track down the source. In contrast, chapter 8 on the biological applications of diamond, written by J. P. Lin, has the form of a standard technical review paper including a 40-entry bibliography

It is not easy to decide what to make of this book and what to recommend to the perspective reader. It is a jumble of fascinating information, wild speculation, and a considerable leavening of mistakes, the latter ranging from the grammatical to the seriously factual. As an example (p. 60), "Most kimberlite pipes were extruded about 100 million years ago, but the diamonds they carry could have been formed even 30 billion years earlier." Given that the age of the Earth is 4.5 billion years and the age of the Universe 13-15 billion years, that statement is to be seriously doubted. 